\title{
A MAXIMUM PRINCIPLE FOR $n$-METAHARMONIC FUNCTIONS
}

\author{
SHUI-NEE CHOW ${ }^{1}$ AND D. R. DUNNINGER
}

ABSTRACT. A class of $n$-metaharmonic functions is shown to satisfy the inequality, $|u(x)| \leqq k\left|u\left(x_{0}\right)\right|$, where $x$ is an arbitrary point in a domain $D, x_{0}$ is some fixed point on the boundary of $D$, and $k$ is a constant.

1. Consider a differential equation of the form

$$
L u \equiv \Delta^{n} u+a_{n-1} \Delta^{n-1} u+\cdots+a_{0} u=0
$$

where $a_{0}, \cdots, a_{n-1}$ are real constants, $\Delta$ is the Laplace operator in Euclidean space of $p$ dimensions $E^{p}$ and $\Delta^{m} \equiv \Delta\left(\Delta^{m-1}\right), \Delta^{0} \equiv I$. Points in $E^{p}$ are denoted by $x=\left(x_{1}, \cdots, x_{p}\right)$. Let $D$ be a bounded domain in $E^{p}$ with a sufficiently smooth boundary $\partial D$. A function $u$ of class $C(\bar{D}) \cap$ $C^{2 n}(D)$ and satisfying (1) in $D$ is called an $n$-metaharmonic function or regular solution [1]. Associated with (1) is the following characteristic equation

$$
f(\lambda) \equiv \lambda^{n}+a_{n-1} \lambda^{n-1}+\cdots+a_{0}=0 .
$$

The main purpose of this paper is to prove the following maximum principle.

THEOREM 1. Suppose the roots $\lambda_{i}$ of (2) satisfy both of the conditions:

(a) $\operatorname{Re} \lambda_{i} \geqq 0$.

(b) If $\operatorname{Re} \lambda_{i}=0$, then $\lambda_{i}$ is a simple root.

If $u$ is a regular solution of (1) satisfying

$$
\Delta u=\Delta^{2} u=\cdots=\Delta^{n-1} u=0 \text { on } \partial D
$$

then there exists a positive constant $k$ and $a$ point $x_{0} \in \partial D$ such that

$$
|u(x)| \leqq k\left|u\left(x_{0}\right)\right| \text { for all } x \in \bar{D} .
$$

Received by the editors March 9, 1973 and, in revised form, July 13, 1973.

AMS (MOS) subject classifications (1970). Primary 35J40, 35B45; Secondary 34D20.

Key words and phrases. $n$-metaharmonic functions, maximum principle, Liapunov stability theory.

${ }^{1}$ Supported under NSF Grant GP-31123X.

(C) American Mathematical Society 1974 
Proof. Let $u$ be a regular solution of (1). Then the $n$ functions $u_{1}=u, u_{2}=\Delta u, \cdots, u_{n}=\Delta^{n-1} u$ satisfy the vector equation

$$
\Delta U=-A U
$$

where

$$
U=\left(\begin{array}{c}
u_{1} \\
u_{2} \\
\cdot \\
\cdot \\
u_{n}
\end{array}\right) \quad A=\left(\begin{array}{rrrrr}
0 & -1 & 0 & \cdots & 0 \\
0 & 0 & -1 & \cdots & 0 \\
\cdot & & & & \\
\cdot & & & & \\
\cdot & & & & \\
0 & 0 & 0 & \cdots & -1 \\
a_{0} & a_{1} & a_{2} & \cdots & a_{n-1}
\end{array}\right)
$$

Note that $-A$ is the companion matrix of the polynomial $f(\lambda)$ and consequently the eigenvalues $\mu_{i}$ of $A$ are given by $\mu_{i}=-\lambda_{i}$ and satisfy both of the conditions:

(a') $\operatorname{Re} \mu_{i} \leqq 0$.

(b') If $\operatorname{Re} \mu_{i}=0$, then $\mu_{i}$ is simple.

According to a well-known result in Liapunov stability theory [2, Theorem $4^{*}$, p. 183] there exists a positive definite matrix $B$ and a positive semidefinite matrix $C$ satisfying

$$
A^{T} B+B A=-C
$$

where " $T$ " denotes the transpose. Defining

$$
V=U^{T} B U
$$

and applying the Laplacian to (7) gives

$$
\Delta V=(\Delta U)^{T} B U+U^{T} B(\Delta U)+2 \sum_{i=1}^{p}\left(U_{x_{i}}\right)^{T} B U_{x_{i}} .
$$

Substituting (5) into (8), using (6), and recalling that $B$ is positive definite and $C$ is positive semidefinite, we find that

$$
\Delta V \geqq-U^{T}\left(A^{T} B+B A\right) U=U^{T} C U \geqq 0 .
$$

According to the maximum principle for subharmonic functions, there exists a point $x_{0} \in \partial D$ such that

$$
V\left(x_{0}\right) \geqq V(x)
$$

for all $x \in \bar{D}$. Since $B$ is positive definite it follows that the diagonal elements $b_{i i}$ of $B$ are positive and moreover that there exists a positive constant $K$ (in fact the lowest eigenvalue of $B$ ) such that $U^{T} B U \geqq K U^{T} U$. 
In view of these remarks and the fact that $u$ satisfies the boundary conditions (3), the inequality in (9) becomes

$$
b_{11} u^{2}\left(x_{0}\right) \geqq K U^{T} U \geqq K u^{2}(x)
$$

and consequently, $|u(x)| \leqq k\left|u\left(x_{0}\right)\right|$ for all $x \in \bar{D}$, where $k=\left(b_{11} / K\right)^{1 / 2}$.

REMARKS.

1. If instead of (3) we prescribe the boundary conditions

$$
u=\Delta u=\cdots=\Delta^{i-1} u=\Delta^{i+1} u=\cdots=\Delta^{n-1} u=0
$$

where $i$ is a given integer such that $0<i \leqq n-1$, then it can be shown, similarly, that there exist an $x_{0} \in \partial D$ and a positive constant $k_{i}$ such that $\left|\Delta^{i} u(x)\right| \leqq k_{i}\left|\Delta^{i} u\left(x_{0}\right)\right|$ for all $x \in \bar{D}$.

2. As a consequence of the inequality (4), the usual type of uniqueness and continuous dependence theorems follow. However, we shall not pursue this point here.

2. Examples.

1. For $n=2$, consider

$$
\begin{aligned}
\Delta^{2} u+a_{1} \Delta u+a_{0} u & =0 & & \text { in } D, \\
\Delta u & =0 & & \text { on } \partial D .
\end{aligned}
$$

The associated characteristic equation $\lambda^{2}+a_{1} \lambda+a_{0}=0$ has roots

$$
\lambda_{1}=\left(-a_{1}+\left(a_{1}^{2}-4 x_{0}\right)^{1 / 2}\right) / 2, \quad \lambda_{2}=\left(-a_{1}-\left(a_{1}^{2}-4 a_{0}\right)^{1 / 2}\right) / 2 .
$$

Hence, the inequality (4) is valid provided one of the following conditions is satisfied:

(i) $a_{1}^{2}-4 a_{0} \leqq 0, \quad a_{1}<0$,

(ii) $a_{1}^{2}-4 a_{0}>0, \quad a_{1}<0, \quad a_{0}>0$,

(iii) $a_{1}=0, \quad a_{0}>0$.

We note that this result was obtained in [3] under condition (iii).

The inequality (4) is easily shown to be false, in general, if $a_{1}>0$. In fact, if we let $D$ denote the region $0<x<\pi, 0<y<\pi$, then the problem

$$
\begin{aligned}
\Delta^{2} u+4 \Delta u+4 u=0 & \text { in } D, \\
u=\Delta u=0 & \text { on } \partial D,
\end{aligned}
$$

has as a solution $u=\sin x \sin y$, for which (4) does not hold.

2 . For arbitrary $n$, consider

$$
\begin{aligned}
(\Delta-a)^{n} u=0 & & \text { in } D, \\
\Delta u=\Delta^{2} u=\cdots=\Delta^{n-1} u=0 & & \text { on } \partial D .
\end{aligned}
$$


The associated characteristic equation has only one distinct root, namely $\lambda=a$. Hence if $a>0$ then inequality (4) is valid. Moreover, the counterexample used in Example 1 shows that inequality (4) is false if $a<0$.

3. Consider the following equation

$$
\Delta^{n} u+a_{0} u=0 \text { in } D .
$$

Since the $n$th roots of 1 or -1 , for $n \geqq 3$, do not satisfy either of the conditions (a) or (b) in Theorem 1, it appears that the inequality (4) is not valid for solutions of (11). However, this is still an open question.

3. Returning to Example 1, it is easily verified that in case (iii) the quadratic expression (7) takes the form

$$
V=a_{0} u^{2}+(\Delta u)^{2} .
$$

By generalizing this expression slightly we are in a position to obtain a maximum principle for the following equation

$$
\Delta^{2} u+a_{0}(x) u=0 \text { in } D
$$

where $a_{0}(x)>0$, is of class $C(\bar{D}) \cap C^{2}(D)$. Specifically we set

$$
V=\alpha(x) u^{2}+\beta(x)(\Delta u)^{2}
$$

where $\alpha$ and $\beta$ will be determined later. Applying the Laplacian to (13) and making use of (12) gives

Choosing

$$
\Delta V=u^{2} \Delta \alpha+2 \alpha|\operatorname{grad} u|^{2}+4 u \operatorname{grad} u \cdot \operatorname{grad} \alpha
$$

$$
\begin{aligned}
& +(\Delta u)^{2} \Delta \beta+2 \beta|\operatorname{grad} \Delta u|^{2}+4 \Delta u \operatorname{grad} \Delta u \cdot \operatorname{grad} \beta \\
& +2 u \Delta u\left(\alpha-\beta a_{0}\right) .
\end{aligned}
$$

and setting

$$
\alpha>0, \quad \beta>0,
$$

in (14), and simplifying, we obtain

$$
\begin{aligned}
\Delta V \geqq & u^{2}\left[\Delta \alpha-(2 / \alpha)|\operatorname{grad} \alpha|^{2}\right]+(\Delta u)^{2}\left[\Delta \beta-(2 / \beta)|\operatorname{grad} \beta|^{2}\right] \\
& +2 \alpha|\operatorname{grad} u+(u / \alpha) \operatorname{grad} \alpha|^{2}+2 \beta|\operatorname{grad} \Delta u+(\Delta u / \beta) \operatorname{grad} \beta|^{2} \\
\geqq & u^{2}\left[-\alpha^{2} \Delta(1 / \alpha)\right]+(\Delta u)^{2}\left[-\beta^{2} \Delta(1 / \beta)\right] .
\end{aligned}
$$

Consequently, $V$ will be subharmonic if in addition to (15) and (16), $1 / \alpha$ and $1 / \beta$ are superharmonic, i.e.,

$$
\Delta(1 / \alpha) \leqq 0, \quad \Delta(1 / \beta) \leqq 0 .
$$


Choosing $\alpha=1, \beta=1 / a_{0}$, it follows that (15), (16) and (17) are satisfied provided $a_{0}$ is superharmonic, i.e., $\Delta a_{0} \leqq 0$. Proceeding as in Theorem 1 , we find that if $\Delta u=0$ on $\partial D$, then there exists a point $x_{0} \in \partial D$ such that $|u(x)| \leqq\left|u\left(x_{0}\right)\right|$ for all $x \in \bar{D}$.

Choosing $\alpha=a_{0}, \beta=1$, then (15), (16) and (17) are satisfied provided $1 / a_{0}$ is superharmonic, i.e., $\Delta\left(1 / a_{0}\right) \leqq 0$. Consequently, if $\Delta u=0$ on $\partial D$, we find

$$
|u(x)| \leqq\left(a_{0}\left(x_{0}\right) / a_{0}(x)\right)^{1 / 2}\left|u\left(x_{0}\right)\right| \leqq k^{*}\left|u\left(x_{0}\right)\right|
$$

where $k^{*}=\left(a_{0}\left(x_{0}\right)\right)^{1 / 2} / \min _{\bar{D}}\left(a_{0}(x)\right)^{1 / 2}$.

In summary, we have proved the following:

THEOREM 2. If $a_{0}(x)>0$ belongs to class $C(\bar{D}) \cap C^{2}(D)$ and if

$$
\Delta\left(1 / a_{0}\right) \leqq 0 \text { or } \Delta a_{0} \leqq 0,
$$

then every regular solution of (12), which satisfies $\Delta u=0$ on $\partial D$, satisfies inequality (4).

4. The preceding results carry over with little difficulty to the case in which the Laplacian is replaced throughout by the general elliptic operator

$$
\sum_{i, j=1}^{p} a_{i j}(x) \frac{\partial^{2} u}{\partial x_{i} \partial x_{j}}, \quad a_{i j}=a_{j i},
$$

provided one of the two matrices $\left(a_{i j}\right)$ or $B$ is diagonal.

\section{REFERENCES}

1. I. N. Vekua, New methods for solving elliptic equations, OGIZ, Moscow, 1948; English transl., Series in Appl. Math., vol. 1, North-Holland, Amsterdam; Interscience, New York, 1967. MR 11, 598; 35 \#3243.

2. J. L. Massera, Contributions to stability theory, Ann. of Math. (2) 64 (1956), 182-206. MR 18, 42.

3. D. R. Dunninger, Maximum principles for solutions of some fourth order elliptic equations, J. Math. Anal. Appl. 37 (1972), 655-658.

Department of Mathematics, Michigan State University, East Lansing, MICHIGAN 48823 